\title{
Predictores de sintomatología postraumática aguda y crecimiento post-estrés tras un accidente laboral reciente
}

\author{
Predictors of Acute Posttraumatic Symptoms and Post- \\ Stress Growth After a Recent Work Accident
}

\author{
Felipe E. García ${ }^{1}$ \\ Francisca Miranda Hermosilla ${ }^{2}$ \\ Universidad Santo Tomás, Facultad de Ciencias Sociales y Comunicaciones, Chile
}

Resumen. El propósito de este estudio fue evaluar un modelo predictivo de sintomatología postraumática aguda (SPT) y de crecimiento post-estrés (CPE) en trabajadores que sufrieron un accidente laboral reciente. Se encuestaron 246 trabajadores (23.2\% mujeres) entre los 18 y los 73 años. Se encontró que los principales predictores de la SPT son: severidad subjetiva del evento, afrontamiento religioso negativo, reinterpretación positiva y rumiación de tipos intrusiva, negativa y deliberada. A su vez, los principales predictores de CPE fueron: rumiación deliberada y afrontamiento religioso positivo. Se realizó un análisis de sendero para evaluar un modelo en el cual la rumiación intrusiva y la deliberada cumplen una función mediadora, obteniendo adecuados índices de ajuste. Se plantea la necesidad de contar con un método de evaluación temprana de las respuestas psicológicas tras un accidente.

Palabras clave. Accidente del trabajo, salud ocupacional, crecimiento postraumático, trastorno por estrés agudo, trastorno por estrés postraumático, riesgo psicosocial.

Abstract. The aim of this study is to evaluate a predictive model of acute posttraumatic symptomatology (PTS) and post-stress growth (PSG) in workers who suffered a recent work accident. To this end, 246 workers (23.2\% women) were surveyed, aged between 18 and 73 years. The main predictors of PTS were subjective severity of event, negative religious coping, positive reinterpretation, and intrusive, brooding and deliberate rumination. At the same time, the main predictors of PSG were deliberate rumination and positive religious coping. A path analysis was performed to evaluate a model in which intrusive and deliberate rumination fulfill a mediating function, obtaining adequate goodness-of-fix indices. The need to have a method of early evaluation of psychological responses after an accident is proposed.

Keywords. Occupational health, posttraumatic growth, acute stress disorder, posttraumatic stress disorder, psycho-social risk.

${ }^{1}$ Felipe E. García. Facultad de Ciencias Sociales y Comunicaciones, Universidad Santo Tomás, Concepción, Chile. Dirección Postal: Arturo Prat 855, Concepción, Chile. Código postal: 4061501. E-mail: felipegarciam@yahoo.es

${ }^{2}$ Francisca Miranda. Facultad de Ciencias Sociales y Comunicaciones, Universidad Santo Tomás, Concepción, Chile. E-mail: frmiranda@udec.cl

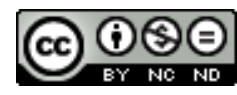

Esta obra está bajo una licencia de Creative Commons Reconocimiento-NoComercial-SinObraDerivada 4.0 Internacional. 


\section{Introducción}

De los múltiples eventos estresantes que pueden afectar a una persona, los accidentes laborales merecen una particular atención debido a su alta frecuencia y a sus consecuencias en la salud física y mental de los afectados (Souto, Oliveira, \& Haag, 2012). Además, generan una pérdida económica, ya sea en forma directa -a través de prestaciones médicas o pago de indemnizaciones-, o indirectas, como días laborales perdidos o menor productividad (Jiménez \& Alvear, 2005).

Los accidentes laborales afectan predominantemente a personas jóvenes, sanas, en plena actividad productiva y a hombres más que a mujeres. Dependiendo de la gravedad de la lesión, sus consecuencias pueden generar cambios importantes en todos los aspectos de su vida, los cuales pueden ser permanentes y definitivos (Asociación Chilena de Seguridad, 2004).

Si bien la prevención primaria de la accidentabilidad ha sido exitosa en Chile, generando una disminución importante y progresiva desde el año 2004 hasta hoy (Superintendencia de Seguridad Social, 2017), la prevención secundaria de las consecuencias en la salud mental en quienes ya han sido afectados por un accidente ha sido menos abordado. Esto por cuanto se ha privilegiado la atención hacia las consecuencias físicas.

En general, las investigaciones acerca de las consecuencias psicológicas de un evento altamente estresante se han centrado en las respuestas psicopatológicas de los afectados (Bonanno, 2004). Entre ellas, las más estudiada han sido el trastorno por estrés agudo (TEA) y el trastorno por estrés postraumático (TEPT). El TEA y el TEPT se caracterizan, según el DSM-IV (American Psychatric Association, 1994), por la presencia de reexperimentación de la vivencia traumática, conductas de evitación y un estado de hipervigilancia tras vivir un acontecimiento que implique la muerte o amenaza para su integridad física o de los demás.

Cuando estas reacciones aparecen en el primer mes que sigue al acontecimiento potencialmente traumático se habla de TEA; cuando persisten más allá de ese tiempo, se habla de TEPT. La presencia de sintomatología asociada a estos trastornos, pero sin el cumplimiento de los criterios necesarios para su diagnóstico, se ha denominado sintomatología postraumática (SPT). Se ha demostrado que el TEA o la sintomatología aguda es un fuerte predictor de TEPT (Brewin, Andrews, \& Valentine, 2000).

En tal sentido, los accidentes laborales constituyen un evento potencialmente traumático capaz de producir TEA (Schönenberg, Reichwald, Domes, Badke, \& Hautzinger, 2008) o TEPT (Ghisi et al., 2013; Gonçalves, Lima, \& Marques, 2006). La respuesta postraumática se origina debido a que el accidente, por lo general, ocurre fuera de los acontecimiento normales y esperables de la vida laboral y que el individuo percibe como abrumador (Vicente-Herrero et al., 2014). La evidencia muestra, además, que hasta un 34\% de las personas que sufren un accidente laboral desarrolla posteriormente TEPT u otros trastornos psiquiátricos; el cual, a su vez, da paso a un importante deterioro en la vida laboral y personal (Nyberg, Stieglitz, Frommberger, \& Berger, 2003).

Por otra parte, existen respuestas más adaptativas tras vivir estos eventos. Una de ellas es crecer o aprender a partir de la experiencia que implica un accidente. A esta posibilidad 
se le ha denominado crecimiento post-estrés o postraumático [CPE], definido como la percepción de cambios positivos que un individuo experimenta como resultado del proceso de lucha ante un evento altamente estresante (Tedeschi \& Calhoun, 1996).

En un meta-análisis realizado por Helgeson, Reynolds y Tomich (2006) se encontró que un mayor CPE se asociaba con un mayor bienestar psicológico. Estos mismos resultados se encuentran en una revisión de estudios longitudinales realizada por Zoellner y Maercker (2006), la cual muestra que el CPE predice menor TEPT en un largo plazo. A su vez, un mayor bienestar percibido por el trabajador tras un accidente laboral parece traer beneficios. Por ejemplo, se relaciona con un menor nivel de accidentabilidad (Kuoppala, Lamminpää, Väänänen-Tomppo, \& Hinkka, 2011; Kirschenbaum, Oigenblick, \& Goldberg, 2000).

De ese modo, resulta importante determinar los factores que contribuyen a la presencia de CPE o SPT agudo tras sufrir un accidente laboral. Se ha señalado, por ejemplo, que la percepción de severidad del evento estresante, es decir la severidad subjetiva, tendría una relación positiva tanto con el desarrollo de SPT (García, Reyes, \& Cova, 2014; Brewin et al., 2009) como del CPE (García et al., 2016; Morris, Shakespeare-Finch, Rieck \& Newbery, 2005).

Otro de los predictores de respuestas posteriores a un evento estresante es la rumiación, un proceso cognitivo consistente en pensar repetidamente sobre las experiencias vividas o sus consecuencias (Calhoun, Cann, \& Tedeschi, 2010). Los dos tipos de rumiación más estudiados en su relación con la salud mental son los de tipo depresivo y los que surgen tras la vivencia de un evento altamente estresante. El primer tipo se divide en rumiación negativa (o brooding) y rumiación reflexiva (Treynor, Gonzalez, \& Nolen-Hoeksema, 2003); mientras que el segundo tipo se subdivide en rumiación intrusiva y deliberada (Cann et al., 2011).

En la rumiación reflexiva, la persona piensa por qué se siente así, analiza las dificultades vividas e intenta entender qué es lo que la lleva a sentir de una determinada forma. En la rumiación negativa, se revive el malestar en forma pasiva (Cova, Rincón, \& Melipillán, 2009). Por otro lado, la rumiación intrusiva se caracteriza por irrupciones involuntarias de pensamientos sobre la experiencia y que no se escoge traer a la mente; la rumiación deliberada se refiere a pensamientos intencionados sobre un evento estresante y pueden enfocarse decididamente en tratar de entender los eventos y sus implicancias (Cann et al., 2011).

En la rumiación negativa se focaliza la atención hacia los síntomas de malestar y está asociada a niveles altos de depresión (Joormann \& Gotlib, 2010). En cambio, la rumiación reflexiva se enfoca en la resolución de los problemas, asociándose a mayores posibilidades de recuperación (Arditte \& Joormann, 2011). Se ha observado, a su vez, que tanto la rumiación deliberada como la intrusiva se activan cuando la persona percibe un evento como estresante y, por ello, en algunos estudios ambas se relacionan positivamente tanto con la SPT como con el CPE, aunque con distinta fuerza (Alzugaray, García, Reyes, \& Álvarez, 2015; Danhauer et al., 2013; García, Duque, \& Cova, 2017; O’Connor, 2017).

Sin embargo, la relación más consistente se ha encontrado entre la rumiación deliberada y CPE (Morris, \& Shakespeare-Finch, 2011; Stockton, Hunt, \& Joseph, 2011) y entre la 
rumiación intrusiva y SPT (Alzugaray et al., 2015). Asimismo, se ha observado que la rumiación deliberada media la relación entre CPE y otras variables como la severidad subjetiva, la rumiación negativa y la rumiación intrusiva (García, Cova, Rincón, \& Vázquez, 2015; García et al., 2016). Por otro lado, la rumiación intrusiva también mediaría la relación entre la severidad del trauma y la SPT (Morris \& Shakespeare-Finch, 2011).

Otro grupo de variables que han sido estudiadas en su relación con las consecuencias, tras la exposición a un evento altamente estresante, son las estrategias de afrontamiento, referidas a los procesos cognitivos y conductuales que se desarrollan para manejar las demandas específicas externas o internas que sobrepasan los recursos del individuo (Lazarus, \& Folkman, 1984). Al respecto, en una revisión de 39 publicaciones, se encontró que el CPE se asoció a estrategias de afrontamiento centradas en el problema y reinterpretación positiva (Linley, \& Joseph, 2004). Además, Rajandram, Jenewein, McGrath y Zwahlen (2011) realizaron un meta-análisis en el que encontraron que el afrontamiento centrado en el problema, búsqueda de apoyo social, afrontamiento religioso y reinterpretación positiva son importantes predictores del CPE, lo que fue refrendado por un estudio realizado en Chile con estudiantes universitarios (Avilés, Cova, Bustos, \& García, 2014).

En el caso específico del afrontamiento religioso, hay evidencia de que la religiosidad permite hacer frente a las secuelas de un evento altamente estresante o potencialmente traumático; por ejemplo, ayudando a las personas a meditar de manera constructiva, para desarrollar resultados positivos tras el evento (O’Connor, 2017). A su vez, las experiencias estresantes o traumáticas pueden conducir a una profundización de la religiosidad (Shaw, Joseph, \& Linley, 2007). Los meta-análisis de Helgeson et al. (2006) y de Prati y Pietrantoni (2009) identifican a la religiosidad como uno de los factores que más influyen en el desarrollo del CPE.

Pargament, Smith, Koenig y Perez (1998) distinguen entre el afrontamiento religioso positivo y el negativo; el primero se vincula al apoyo espiritual, el perdón, la purificación religiosa y la búsqueda de ayuda de miembros de la iglesia. El segundo, en cambio, hace referencia a una apreciación religiosa punitiva, el descontento espiritual y el descontento religioso interpersonal. Los estudios muestran una relación distinta del afrontamiento religioso positivo y negativo con las respuestas psicológicas tras un evento altamente estresante. Por ejemplo, se ha observado que quienes tienen una visión religiosa negativa presentan peor salud física, peor calidad de vida y una mayor depresión (Koenig, Pargament, \& Nielsen, 1988); en cambio, la visión religiosa positiva se relacionaba a un mayor CPE (Bosson, Kelley, \& Jones, 2012; García, Páez, Cartes, Neira, \& Reyes, 2014) o un mayor bienestar psicológico (Gholamzadeh, Hamid, Basri, Sharif, \& Ibrahim, 2014).

Frente a estos antecedentes, el propósito de este estudio fue evaluar un modelo predictivo que incluye distintos tipos de rumiación y de estrategias de afrontamiento, además de la severidad subjetiva del evento, en la predicción diferencial de sintomatología postraumática aguda y crecimiento post-estrés en personas que han sufrido un accidente laboral en el último mes. En este modelo, en coherencia con la evidencia disponible, la rumiación deliberada cumple una función mediadora entre las otras variables y el CPE y la rumiación intrusiva cumple una función mediadora entre las otras variables y el SPT (ver figura 1). 


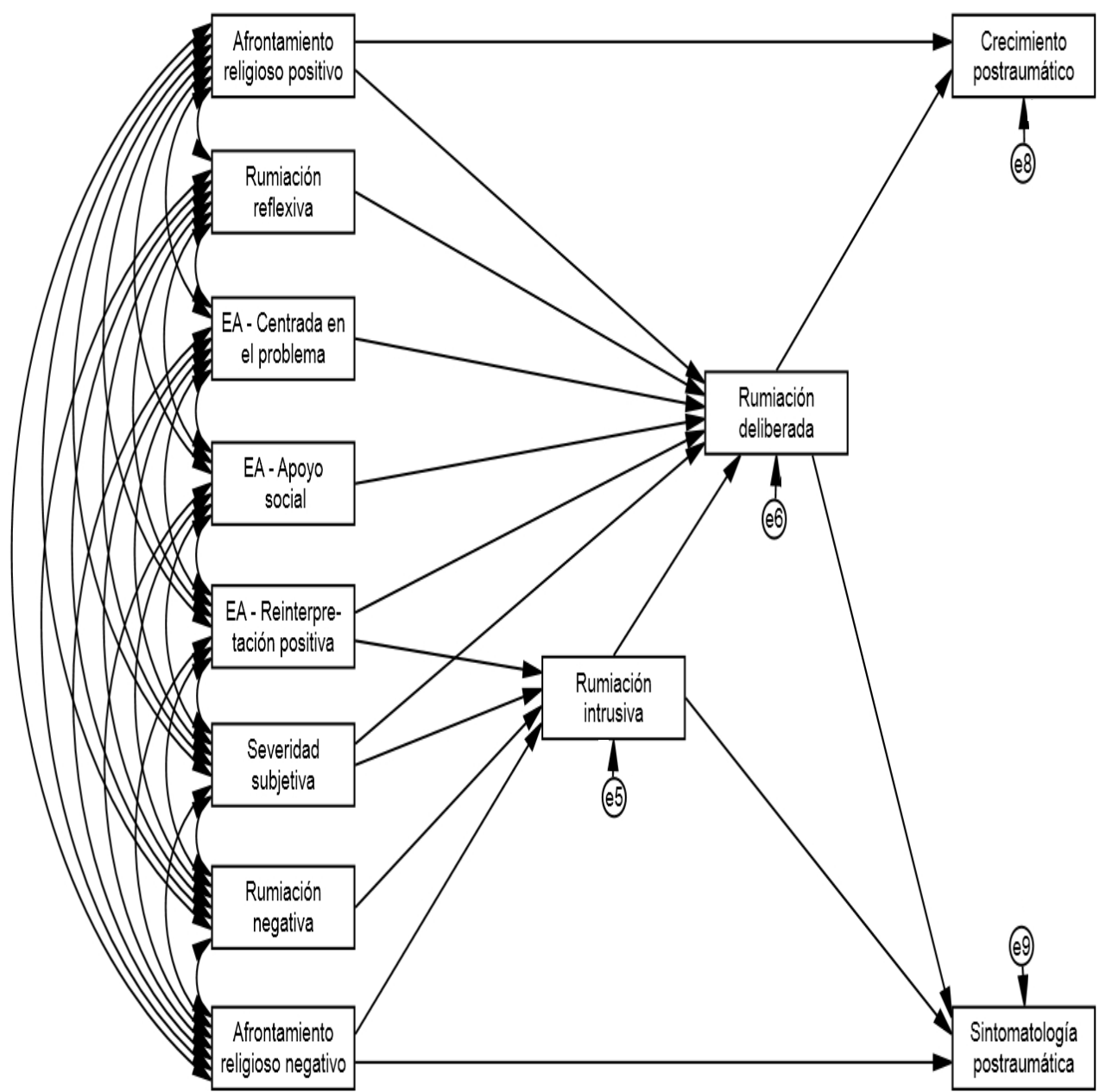

Figura 1. Modelo de sendero hipotetizado

\section{Método}

\section{Diseño}

Se utilizó un diseño cuantitativo de tipo descriptivo y correlacional. Las mediciones se llevaron a cabo en un solo momento, por lo que se trata de un estudio transversal.

\section{Participantes}

Se Participaron 246 trabajadores de la provincia de Concepción, Chile, que sufrieron un accidente laboral en el último mes antes de la evaluación y que haya implicado al menos 10 días de licencia médica. De ellos, 57 fueron mujeres (23.2\%) y 189 hombres $(76.8 \%)$. La media total de edad fue de 39.37 años $(D T=11.86)$ con un rango desde los 18 a los 73 años. 


\section{Instrumentos}

Escala de severidad subjetiva del evento (SSE). Es una escala para detectar la severidad con la que se percibe el evento estresante (Alzugaray et al., 2015). Consta de tres ítems que evalúan a) si la persona percibe que el evento alteró su vida, b) si la persona percibe el evento como traumático y c) si percibe como grave lo que le ha pasado. Se responde en una escala Likert en un rango que va de 0 a 4 puntos. En el estudio de Alzugaray et al., esta escala mostró una consistencia interna adecuada $(\alpha=.84)$.

Escala de Respuesta Rumiativa (RRS). Fue propuesta por Treynor et al. (2003) y validada en Chile por Cova, Rincón y Melipillán (2007) para evaluar la rumiación reflexiva y negativa. Consta de 14 ítems que se responden en una escala Likert que va desde 0 (casi nunca) a 3 (casi siempre). En el estudio de Cova et al. (2007) se observaron satisfactorios indicadores de confiabilidad, tanto en reflexión $(\alpha=.75)$ como en rumiación negativa $(\alpha=.80)$, asî como validez de constructo en la distinción entre las dos formas de rumiación.

Escala de Rumiación Relacionada al Evento (ERRI). Fue creada por Cann et al. (2011) y mide la rumiación intrusiva y la deliberada. Fue validada para su uso en población chilena por Alzugaray et al. (2015), quienes presentaron una versión más breve de 12 ítems: 7 para rumiación intrusiva y 5 para rumiación deliberada. Se responde en una escala Likert desde 0 (casi nunca) a 3 (casi siempre). La versión de Alzugaray et al. obtuvo una consistencia interna adecuada, tanto para rumiación intrusiva $(\alpha=$.91) como para rumiación deliberada $(\alpha=.85)$ y validez de constructo al diferenciar entre ambos factores.

Brief-RCOPE. Fue elaborado por Pargament et al. (1998) para la evaluación del afrontamiento religioso. Fue traducida al español por Rivera-Ledesma y Montero (2005). Tiene dos subescalas de 7 ítems cada una: afrontamiento religioso positivo y su contraparte negativa. Se responde en una escala Likert desde 0 a 3 puntos. Rivera-Ledesma y Montero (2005), a su vez, obtuvieron en población latinoamericana confiabilidad adecuada para las dos dimensiones, además de validez de convergencia con otras escalas de religiosidad.

Brief-COPE. Fue elaborada por Carver (1997) para la medición de las estrategias de afrontamiento. Fue traducida y validada al español por Morán, Landero y González (2010). Para este estudio, se utilizaron las sub-escalas de búsqueda de apoyo instrumental y emocional, para la evaluación del apoyo social (4 ítems), y las subescalas de afrontamiento activo y planificación para la medición del afrontamiento centrado en el problema (4 ítems), pues estas sub-escalas convergen en las dimensiones mencionadas según el análisis factorial desarrollado por Morán et al. Se incluyó también la subescala de reinterpretación positiva ( 2 ítems). Se responden en una escala Likert desde 0 a 3 puntos.

SPRINT-E. Fue creada por Connor y Davidson (2001) para evaluar sintomatología postraumática, según los criterios del DSM-IV. Fue validada en Chile por Leiva-Bianchi y Gallardo (2013). Posee 12 preguntas que se responden en una escala Likert desde 0 (nada) a 3 (mucho). Leiva-Bianchi y Gallardo obtuvieron una confiabilidad alta para esta escala $(\alpha=.92)$.

Inventario de Crecimiento Postraumático, versión breve (PTGI-SF). Fue creado por Cann et al. (2010) y validada para su uso en población chilena por García y Wlodarczyk (2015) para la evaluación del crecimiento post-estrés. Está compuesta de 10 ítems que se responden 
en una escala Likert de seis alternativas, desde 0 (ningún cambio) hasta 5 (un cambio muy importante). En el estudio de García y Wlodarczyk se obtuvo un $\alpha=.94$.

Cuestionario socio-demográfico. Se incluyó un cuestionario con preguntas relativas al género y edad, días desde el accidente y días de licencia.

\section{Procedimiento}

Los participantes se seleccionaron desde un listado de personas accidentadas recientemente, con una licencia médica superior a 10 días y cuya atención médica fue realizada en un hospital especializado en salud laboral de la ciudad de Concepción (población objetivo). Del listado total de 346 personas que cumplieron con los requisitos de inclusión a lo largo de cinco meses, 87 de ellos fueron excluidos debido a que no fueron ubicados en los teléfonos o dirección suministrados por el hospital y 13 de ellos se negaron a responder, por lo que el grupo descendió a 246 personas (población marco), el 71\% del total.

Los participantes fueron encuestados por estudiantes de psicología, previamente capacitados. Los cuestionarios fueron auto-administrados en presencia del encuestador, previa firma de una carta de consentimiento informado en la cual se garantizaba la confidencialidad, resguardo de los datos entregados y participación voluntaria. Este estudio fue aprobado por la Comisión de Ética de la Mutual de Seguridad, Chile (Nº3/15).

\section{Análisis de datos}

En primer lugar, se realizó un análisis de datos descriptivo de las variables independientes y dependientes. Se utilizó la correlación $r$ de Pearson y la Regresión Lineal Múltiple (RLM) para estimar la influencia que tienen las variables predictoras sobre el CPE y la SPT. Finalmente, se utilizó un análisis de sendero para probar el modelo hipotetizado señalado en la figura 1, en el cual la rumiación intrusiva y deliberada cumplen una función mediadora. Para ello, se utilizó el método de estimación de máxima verosimilitud, considerando los siguientes índices y criterios de ajuste (Hu, \& Bentler, 1999; Yu, 2002): a) $\chi^{2}$ : valores no significativos indican un buen ajuste; b) $\chi^{2} / g l$ : un buen ajuste está indicado por valores menores a 2; c) CFI and TLI: un ajuste aceptable está dado por valores $\geq .90$, un buen ajuste está indicado por valores $\geq .95$; d) RMSEA: un ajuste aceptable está indicado por valores $\leq 0.08(90 \% \mathrm{CI} \leq 0.10)$, un buen ajuste está indicado por valores $\leq .05(90 \% \mathrm{CI}$ $\leq .08)$. Para todos estos análisis se utilizó el software estadístico SSPS Statistics 23 y el Amos v18 de IBM.

\section{Resultados}

En primer lugar, se calcularon los estadísticos descriptivos de las variables estudiadas. Se observan unos coeficientes de asimetría y de curtosis dentro del rango aceptable para considerar que cada variable se distribuye normalmente. En cuanto a la consistencia interna de las escalas, establecida mediante el coeficiente $\alpha$ de Cronbach, éstas oscilaron entre .65 y .93 , lo cual permite afirmar que todas las escalas utilizadas son suficientemente confiables. En la tabla 1 se pueden observar además los otros estadísticos descriptivos.

Se realiza un análisis de correlación bivariada entre las variables, obteniendo los resultados que se detallan en la tabla 2. En ella se observa que la SPT se correlaciona significativamente 
Tabla 1

Estadísticos descriptivos y consistencia interna (alfa de Cronbach) de las variables de estudio $(\mathrm{N}=246)$

\begin{tabular}{lccccc}
\hline & Mín & Máx & $M$ & $D T$ & $\alpha$ \\
& & & & & \\
\hline Severidad subjetiva & 0 & 15 & 6.43 & 3.49 & .87 \\
Rumiación - Negativa & 0 & 21 & 7.59 & 5.25 & .85 \\
Rumiación - Reflexiva & 0 & 20 & 7.41 & 4.63 & .78 \\
Rumiación - Deliberada & 0 & 15 & 6.12 & 4.05 & .81 \\
Rumiación - Intrusiva & 0 & 21 & 6.83 & 6.34 & .93 \\
EA - Reinterpretación positiva & 0 & 6 & 2.78 & 1.75 & .65 \\
EA - Apoyo social & 0 & 12 & 5.92 & 3.03 & .71 \\
EA - Centrado en el problema & 0 & 12 & 5.86 & 3.08 & .71 \\
Afrontamiento religioso positivo & 0 & 21 & 7.14 & 6.80 & .93 \\
Afrontamiento religioso negativo & 0 & 15 & 1.40 & 2.56 & .72 \\
Sintomatología postraumática & 0 & 33 & 9.92 & 8.25 & .90 \\
Crecimiento post-estrés & 0 & 50 & 21.26 & 12.93 & .89 \\
\hline
\end{tabular}

Nota. EA = Estrategia de afrontamiento

con todas las variables, a excepción de reinterpretación positiva; la mayor correlación la tiene con la rumiación intrusiva $(r=.77)$. Por otra parte, se observa que el CPE también correlaciona positivamente con todas las variables, obteniendo la correlación más fuerte con el afrontamiento religioso positivo $(r=.56)$.

A continuación, se realizaron las RLM para conocer la influencia de las variables predictoras sobre la SPT y CPE (ver tabla 3). En primer término, se evaluó la influencia sobre la SPT, los resultados muestran un valor $F_{(10,228)}=42.546, p>.001$, con un $R^{2}=$ $.65\left(R^{2} a d j=.64\right)$, las influencias significativas fueron la rumiación intrusiva $(\beta=.46)$, la rumiación negativa $(\beta=.18)$, rumiación deliberada $(\beta=.13)$, la severidad subjetiva $(\beta=$ $.12)$, el afrontamiento religioso negativo $(\beta=.10)$, además de la reinterpretación positiva como predictor negativo $(\beta=-.11)$.

En cuanto al CPE, los resultados muestran un valor $F_{(10,228)}=18.026, p>.01$ con un $R^{2}=$ $.44\left(R^{2} a d j=.42\right)$. Las influencias significativas fueron el afrontamiento religioso positivo $(\beta=.37)$ y la rumiación deliberada $(\beta=.23)$.

Como último paso, se puso a prueba el análisis de sendero hipotetizado en la figura $1 \mathrm{a}$ través del análisis de sendero. Se obtuvieron los siguientes índices de ajuste: a) $\chi^{2}=49.00$, $p<.001$; b) $\chi^{2} / g l=2.23$; c) CFI $=.98$; c) TLI $=.94$; d) RMSEA $=.07$ (IC $\left.=0.04-0.10\right)$. De acuerdo con los parámetros ya señalados, estos índices se consideraron saisfactorios, lo que implica que los datos del estudio concuerdan con el modelo hipotetizado. 


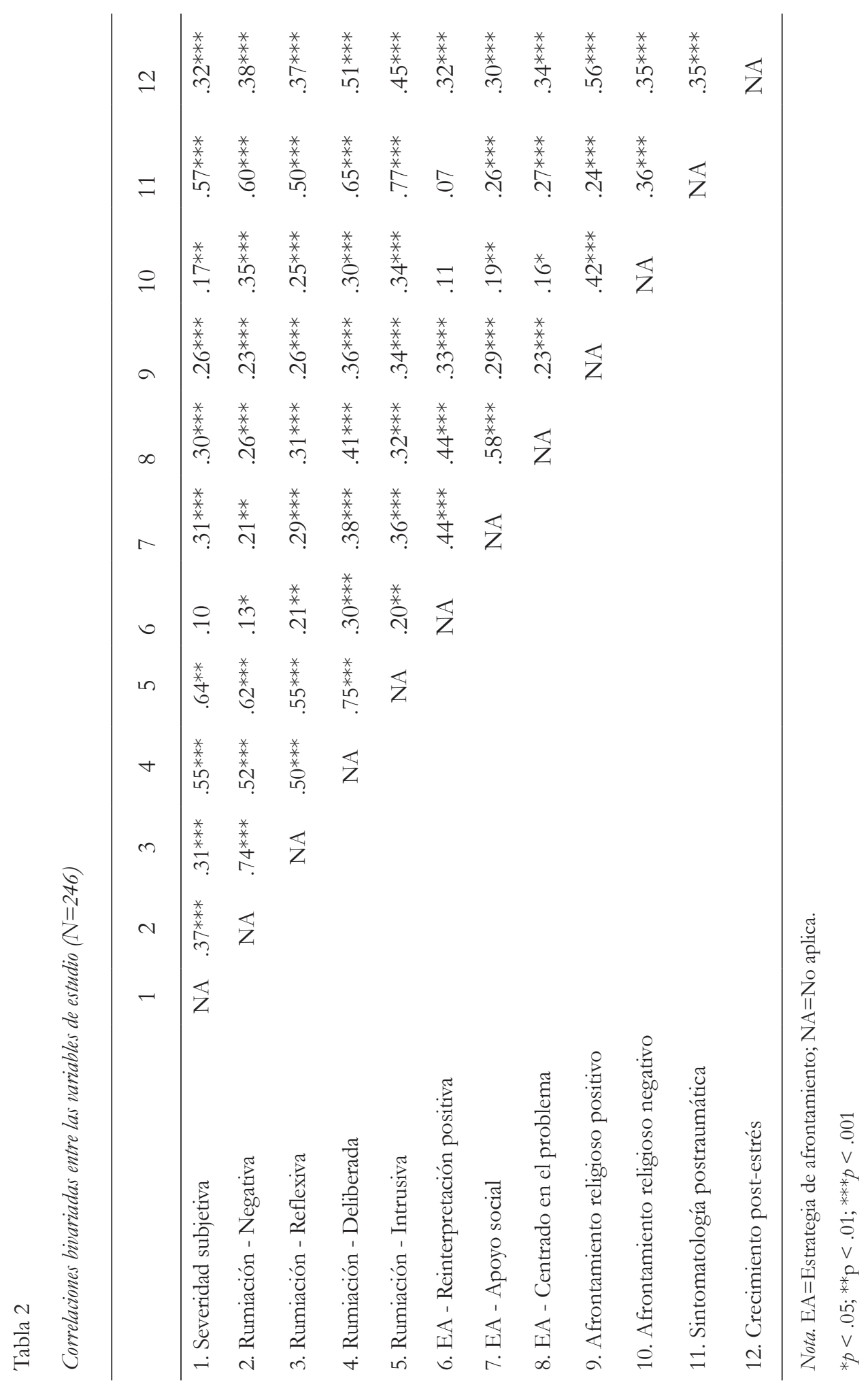

Actualidades en Psicología, 33(126), enero-junio 2019, 117-135 
Tabla 3

Regresión lineal múltiple de las variables de estudio sobre la sintomatología postraumática y el crecimiento post-estrés $(\mathrm{N}=246)$

\begin{tabular}{lccccccc}
\hline & \multicolumn{3}{c}{$\begin{array}{c}\text { Sintomatología } \\
\text { postraumática }\end{array}$} & \multicolumn{3}{c}{ Crecimiento post-estrés } \\
& $\beta$ & $t$ & $p$ & $\beta$ & $t$ & $p$ \\
(Constante) & & 1.023 & .307 & & 3.013 & .003 \\
Severidad subjetiva & .12 & 2.313 & .022 & -.02 & -0.265 & .791 \\
Rumiación - Negativa & .18 & 2.808 & .005 & .08 & 1.000 & .318 \\
Rumiación - Reflexiva & .00 & 0.075 & .940 & .04 & 0.544 & .587 \\
Rumiación - Deliberada & .13 & 2.082 & .038 & .23 & 2.869 & .005 \\
Rumiación - Intrusiva & .46 & 6.568 & .001 & .02 & 0.207 & .836 \\
EA - Reinterpretación positiva & -.11 & -2.373 & .018 & .06 & 1.065 & .288 \\
EA - Apoyo social & -.01 & -0.176 & .860 & -.03 & -0.495 & .621 \\
EA - Centrado en el problema & .04 & 0.859 & .391 & .11 & 1.651 & .100 \\
Afrontamiento religioso positivo & -.05 & -1.119 & .264 & .37 & 6.358 & .001 \\
Afrontamiento religioso negativo & .10 & 2.188 & .030 & .07 & 1.293 & .197 \\
\hline
\end{tabular}

Nota. $\mathrm{EA}=$ Estrategia de afrontamiento; $\mathrm{AR}=$ Afrontamiento religioso.

Se realiza una reespecificación del modelo eliminando de este las relaciones no significativas: apoyo social $\rightarrow$ rumiación deliberada, afrontamiento religioso positivo $\rightarrow$ rumiación deliberada y reintepretación positiva $\rightarrow$ rumiación intrusiva. Los índices de modificación sugirieron agregar la relación reinterpretación positiva $\rightarrow$ sintomatología postraumática. Ese modelo reespecificado, que se muestra en la figura 2, obtuvo los siguientes índices de bondad de ajuste: : a) $\chi^{2}=48.96, p<.002$; b) $\chi^{2} / g l=2.04$; c) CFI $=.98 ;$ c) TLI $=.95 ; \mathrm{d})$ RMSEA $=.07$ (IC $=.04 / .09)$, los que implican un leve mejoramiento respecto al modelo inicial.

\section{Discusión y Conclusiones}

Este estudio pretendía evaluar un modelo predictivo de sintomatología postraumática aguda y crecimiento post-estrés en trabajadores que habían sufrido recientemente un accidente laboral. Para ello, se eligieron como predictores aquellas que según la literatura disponible tenían una relación más fuerte con las variables de resultado indicadas. Esto explica por qué las correlaciones entre las variables fueran en todos los casos significativas, a excepción de la reinterpretación positiva, que mostró no tener una relación bivariada con la SPT. El resto de las variables, la rumiación negativa, reflexiva, intrusiva y deliberada, 


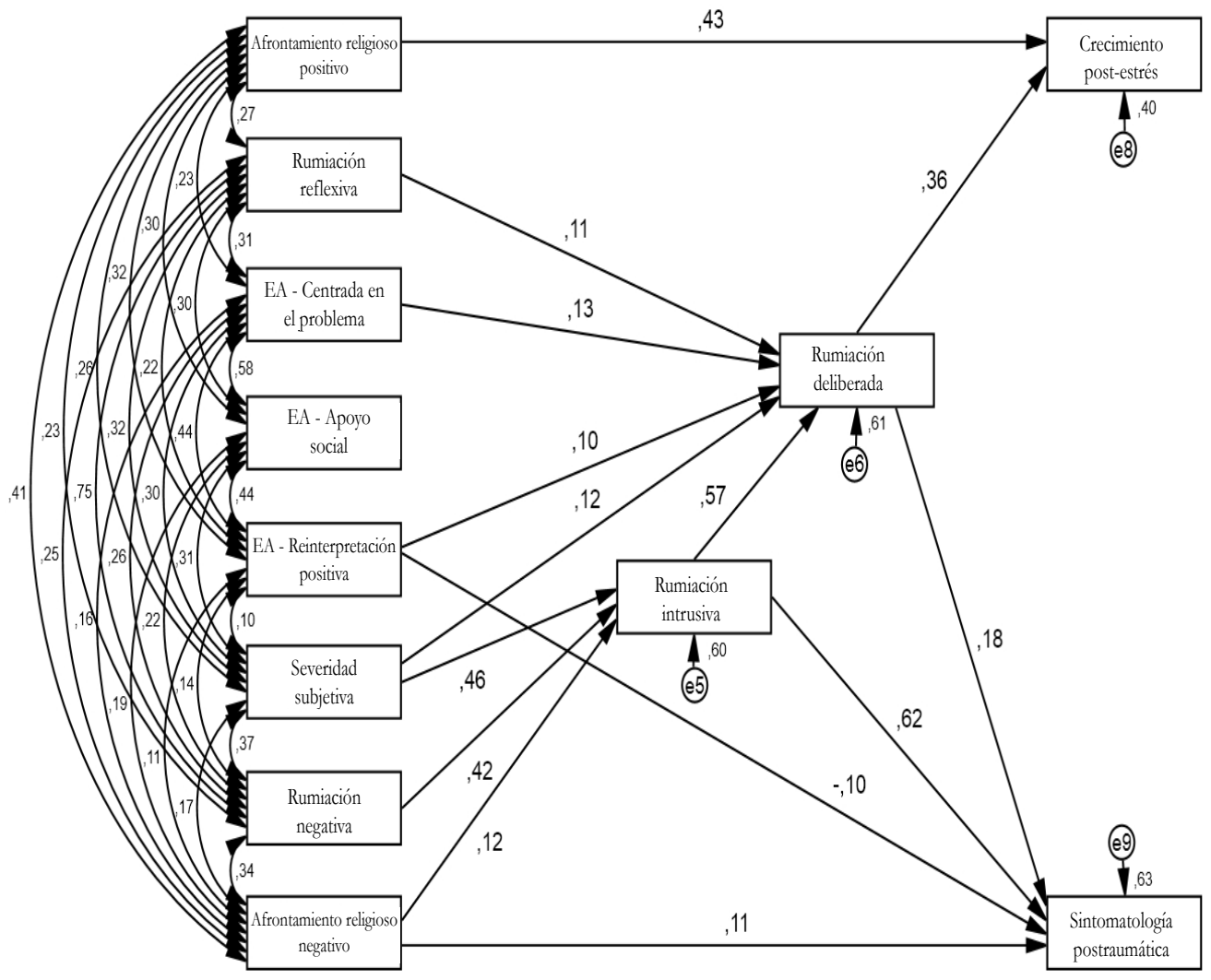

Figura 2. Modelo de sendero reespecificado. Los pesos están estandarizados.

las estrategias de afrontamiento de búsqueda de apoyo social, centrada en el problema, religioso positivo y religioso negativo mostraron correlaciones bivariadas directas y significativas tanto con SPT como con CPE. Incluso, entre la misma SPT y CPE, se observa una correlación directa significativa.

La relación directa entre SPT y CPE y la presencia de relaciones directas con las mismas variables independientes no es de extrañar. Hay una abrumadora cantidad de literatura que da cuenta de esta relación y es lo que ha hecho cuestionar a algunos investigadores sobre la supuesta naturaleza adaptativa del CPE (ver Vázquez, Castilla, \& Hervás, 2009), considerada una variable más positiva y relacionada con el bienestar. Sin embargo, dicha relación se explica por el hecho de que ambas respuestas se desencadenan tras la percepción de un evento como severo (García, Jaramillo, Martínez, Valenzuela, \& Cova, 2014; Hafstad, Gil-Rivas, Kilmer, \& Raeder, 2010; Kilmer, \& Gil-Rivas, 2010); es decir, sin un evento estresante que lo preceda, no surgirían ni la SPT ni el CPE. 
De ahí que se hizo necesario estudiar la influencia diferencial de las variables predictoras sobre una u otra respuesta, con el fin de distinguir qué estrategias llevan al SPT y cuáles al $\mathrm{CPE}$, a través de la RLM. Al realizar este ejercicio, surgen las diferencias esperadas. En este caso, la severidad subjetiva del evento, la rumiación negativa, la rumiación deliberada, la rumiación intrusiva y el afrontamiento religioso negativo se constituyen como predictores significativos directos de la SPT. A su vez, la reinterpretación positiva aparece como un predictor inverso de la misma. Por otro lado, la rumiación deliberada y el afrontamiento religioso positivo ejercen una influencia significativa directa sobre el CPE.

Los resultados indicados son coherentes con la literatura disponible. Por ejemplo, aquellos estudios que buscan determinar la influencia diferencial de los procesos de rumiación en la SPT y CPE muestran que la rumiación negativa e intrusiva serían un factor de vulnerabilidad en el desarrollo y mantenimiento de la SPT, en cambio la rumiación deliberada tendrían una influencia más fuerte sobre el CPE que sobre la SPT (Alzugaray et al., 2015; García et al., 2015; Soo, \& Sherman, 2015; Triplett, Tedeschi, Cann, Calhoun, \& Reeve, 2012). La rumiación negativa e intrusiva actuarían incluso inhibiendo el desarrollo de CPE (Stockton et al., 2011; Taku, Cann, Tedeschi, \& Calhoun, 2009).

En cuanto a las estrategias de afrontamiento, la búsqueda de apoyo social, centrarse en el problema y la reinterpretación positiva no mostraron influencia sobre CPE y solo la última influye de modo inverso sobre la SPT, lo que luego se ve reafirmado en el análisis de sendero. La reinterpretación positiva es considerada una estrategia activa de tipo cognitiva, que permite revalorar experiencias negativas estresantes, específicamente la persona reconstruye una situación estresante en términos positivos, lo que le permite reducir el estrés (Carver, 1997).

$\mathrm{El}$ afrontamiento religioso es otra variable que influye en forma diferencial sobre el CPE y la SPT. En este caso, el afrontamiento positivo influye sobre el CPE y el negativo sobre la SPT. Esto también concuerda con investigaciones previas que muestran una relación distinta de ambos tipos de afrontamiento con las respuestas psicológicas tras un evento estresante o potencialmente traumático (Pargament, Koenig, Tarakeshwar, \& Hahn, 2004). El afrontamiento religioso negativo implicaría ver a una deidad o la religión en términos punitivos o lejanos (Bradley, Schwartz, \& Kaslow, 2005); cuestionar la bondad de dicha deidad (McConnell, Pargament, Ellison, \& Flannelly, 2006) o presentar dudas sobre la existencia de un ser que protege a la gente buena de eventos negativos (Pargament, 1997), lo que contribuye a aumentar la SPT. A su vez, la influencia del afrontamiento religioso positivo sobre el CPE, corrobora el resultado de otros estudios (García, Páez et al., 2014) e implica que este afrontamiento aportaría a encontrar un mayor sentido de la vida proporcionado un marco de significado que ayudar a comprender y aceptar la naturaleza de los eventos estresantes y sus consecuencias (Shaw et al., 2005).

La evaluación del modelo de sendero a través de ecuaciones estructurales construido para comparar la influencia de las variables predictoras sobre ambas variables dependientes al mismo tiempo -con la rumiación intrusiva y deliberada como mediadores-, refuerza la idea de que existen vías diferenciales. Por un lado, reafirma que la rumiación deliberada participa tanto en la SPT como en el CPE, un fenómeno que a Leal, Carmona y Ferrer (2016) no 
les parece inesperado, pues el CPE es una respuesta relacionada con la aparición de experiencias altamente estresantes o potencialmente traumáticas, lo que implica la necesidad de resignificar dicha experiencia; sin embargo, la experiencia de crecimiento no necesariamente reemplaza la experiencia del estrés, más bien coexisten, tal como lo plantea el modelo original de Tedeschi y Calhoun (2004).

De ese modo, la rumiación deliberada, probablemente, también contribuye a mantener el foco en el evento estresante. Para Leal et al. (2016), el punto crítico no es que la rumiación deliberada suprima el estrés, sino que el paso de la rumiación intrusiva hacia la rumiación deliberada posibilite el crecimiento psicológico, hecho que también se puede observar en el modelo evaluado en el presente estudio. De ahí el rol mediador de la rumiación deliberada, pues es a través de ella que la rumiación intrusiva puede dar cuenta de experiencias de crecimiento, lo que coincide con lo encontrado en estudios previos (García et al., 2016).

Otro aspecto interesante del modelo de sendero presentado es el rol mediador total o parcial de la rumiación deliberada e intrusiva, entre las estrategias de afrontamiento que las personas utilizan y las respuestas psicológicas relacionadas al evento estresante. Este rol mediador de ambos tipos de rumiación forma parte del modelo teórico de Tedeschi y Calhoun (2004) y ya se había observado en estudios anteriores (Morris, \& ShakespeareFinch, 2011; García et al., 2015, García et al., 2016). Este resultado reafirma la importancia de la elaboración cognitiva tanto del evento mismo como de las acciones que las personas emprenden para manejar la situación, para el desarrollo tanto del SPT como del CPE tras un evento estresante.

El estudio tiene limitaciones que es necesario consignar. La primera de ellas, y quizás la más relevante a la hora de interpretar los datos, es su diseño transversal; pues, si bien se hipotetizan relaciones lineales entre variables predictoras y variables resultado, estas relaciones solo se pueden confirmar en diseños experimentales y/o longitudinales. Los modelos presentados en este artículo conservan, por lo tanto, su carácter hipotético aun cuando constituyen un paso adelante a la hora de establecer los mecanismos psicológicos que conducen ya sea al estrés o al crecimiento. Una segunda limitante es lo reciente del hecho estresante, que impide hablar de estrés postraumático propiamente o de crecimiento como tal, pues para hablar de ello debe transcurrir más tiempo. Sin embargo, la medición de estas variables en forma temprana de igual modo constituye un aporte para evaluar y conocer las respuestas más agudas ante los accidentes laborales y, eventualmente, disponer de acciones más inmediatas para ayudar a los afectados.

\section{Conclusión}

Descubrir qué procesos psicológicos conducen al malestar y cuáles al crecimiento es relevante tanto para detectar la población vulnerable a fin de intervenir tempranamente, así como prevenir la cronicidad del malestar inicial, potenciando el empleo de estrategias de afrontamiento efectivas en esta población, mejorando la calidad de vida de los afectados y disminuyendo los costes asociados, como la pérdida de días de trabajo, licencias médicas, solicitud de atenciones sanitarias, y mayor probabilidad de sufrir un nuevo accidente, entre otras. Se espera que este estudio sea un aporte en esa dirección.

Actualidades en Psicología, 33(126), enero-junio 2019, 117-135 


\section{Referencias}

Alzugaray, C., García, F., Reyes, A., \& Álvarez, R. (2015). Propiedades psicométricas de una versión breve de la escala de rumiación relacionada a un evento en población chilena afectada por eventos altamente estresantes. Ajayu, 13(2), 183-198.

American Psychiatric Association. (1994). Diagnostic and Statistical Manual of Mental Disorders. (4th ed.) Washington, DC: American Psychiatric Press.

Arditte, K. A., \& Joormann, J. (2011). Emotion regulation in depression: Reflection predicts recovery from a major depressive episode. Cognitive Therapy and Research, 35(6), 536-543. doi: 10.1007/s10608-011-9389-4

Asociación Chilena de Seguridad. (2004). Anuario Estadístico 2003. Santiago, Chile: Gerencia de Prevención, ACHS.

Avilés, P., Cova, F., Bustos, C. \& García, F. E. (2014). Afrontamiento y rumiación frente a eventos adversos y crecimiento postraumático en estudiantes universitarios. Liberabit, 20(2), 281-292.

Bonanno, G. A. (2004). Loss, trauma and human resilience: Have we underestimated the human capacity to thrive after extremely aversive events? American Psychologist, 59(1), 20-28. doi: 10.1037/0003-066x.59.1.20

Bosson, J., Kelley, M. L., \& Jones, G. (2012). Deliberate cognitive processing mediates the relation between positive religious coping and posttraumatic growth. Journal of Loss and Trauma, 17(5), 439-451. doi: 10.1080/15325024.2011.650131

Bradley, R., Schwartz, A. C., \& Kaslow, N. J. (2005). Posttraumatic stress disorder symptoms among low-income, African American women with a history of intimate partner violence and suicidal behaviors: Self-esteem, social support, and religious coping. Journal of Traumatic Stress, 18(6), 685-696. doi: 10.1002/jts.20077

Brewin, C. R., Andrews, B., \& Valentine, J. D. (2000). Meta-analysis of risk factors for posttraumatic stress disorder in trauma-exposed adults. Journal of Consulting and Clinical Psychology, 68(5), 748-766. doi: 10.1037/0022-006X.68.5.748

Calhoun, L. G., Cann, A., \& Tedeschi, R. G. (2010). The posttraumatic growth model: Socio-cultural considerations. In T. Weiss \& R. Berger (Eds.), Posttraumatic growth and culturally competent practice: Lessons learned from around the globe (pp. 1-14). Hoboken, NJ: Wiley.

Cann, A., Calhoun, L., Tedeschi, R., Triplett, K., Vishnevsky, T., \& Lindstrom, C. (2011). Assessing posttraumatic cognitive processes: The event related rumination inventory. Anxiety, Stress, \& Coping, 24(2), 137-156. doi: 10.1080/10615806.2010.529901

Carver, C. S. (1997). You want to measure coping but your protocol's too long: Consider the Brief COPE. International Journal of Behavioral Medicine, 4(1), 92-100. doi: 10.1207/s15327558ijbm0401_6 
Connor, K., \& Davidson, J. (2001). SPRINT: A brief global assessment of post-traumatic stress disorder. International Clinical Psychopharmacology, 16(5), 279-284. doi: 10.1097/00004850-200109000-00005

Cova, F., Rincón, P., \& Melipillán, R. (2007). Rumiación y presencia de sintomatología ansiosa y depresiva en adolescentes. Revista Mexicana de Psicología, 24(2), 175-183.

Cova, F., Rincón, P., \& Melipillán, R. (2009). Reflexión, rumiación negativa y desarrollo de sintomatología depresiva en adolescentes de sexo femenino. Terapia Psicológica, 27(2), 155-160. doi: 10.4067/S0718-48082009000200001

Danhauer, S. C., Russell, G. B., Tedeschi, R. G., Jesse, M. T., Vishnevsky, T., Daley, K., ... \& Powell, B. L. (2013). A longitudinal investigation of posttraumatic growth in adult patients undergoing treatment for acute leukemia. Journal of Clinical Psychology in Medical Settings, 20(1), 13-24. doi:10.1007/s10880-012-9304-5

García, F. E., Cova, F., Rincón, P., \& Vázquez, C. (2015). Trauma or growth after a natural disaster? The mediating role of rumination processes. European Journal of Psychotraumathology, 6(1), 1-10. doi: 10.3402/ ejpt.v6.26557

García, F. E., Cova, F., Rincón, P., Vázquez, C., \& Páez, D. (2016). Coping, rumination and posttraumatic growth in people affected by an earthquake. Psicothema, 28(1), 59-65. doi: 10.7334/psicothema2015.100

García, F., Duque, A, \& Cova, F. (2017). The four faces of rumination to stressful events: A psychometric analysis. Psychological Trauma: Theory, Research, Practice, and Police, 9(6), 758-765. doi: 10.1037/tra0000289

García, F. E., Jaramillo, C., Martínez, A. M., Valenzuela, I., \& Cova, F. (2014). Respuestas psicológicas ante un desastre natural: Estrés y crecimiento postraumático. Liberabit, 20(1), 121-130.

García, F., Páez, D., Cartes, G., Neira, H., \& Reyes, A. (2014). Religious coping, social support and subjective severity as predictors of posttraumatic growth in people affected by the earthquake in Chile on 27/F 2010. Religions, 5(4), 1132-1145. doi: $10.3390 / \mathrm{rel} 5041132$

García, F. E., Reyes, A., \& Cova, F. (2014). Severidad del trauma, optimismo, crecimiento postraumático y bienestar en sobrevivientes de un desastre natural. Universitas Psychologica, 13(2), 15-24. doi: 10.11144/javeriana.upsy13-2.stop

García, F., \& Wlodarczyk, A. (2015). Psychometric properties of the Posttraumatic Growth Inventory - Short Form among Chilean adults. Journal of Loss and Trauma, 21(4), 303-314. doi: 10.1080/15325024.2015.1108788

Ghisi, M., Novara, C., Buodo, G., Kimble, M. O., Scozzari, S., Natale, A. D., ... \& Palomba, D. (2013). Psychological distress and post-traumatic symptoms following occupational accidents. Behavioral Sciences, 3(4), 587-600. doi: 10.3390/bs3040587 
Gholamzadeh, S., Hamid, T. A., Basri, H., Sharif, F., \& Ibrahim, R. (2014). Religious coping and psychological well-being among Iranian stroke caregivers. Iranian Journal of Nursing and Midwifery Research, 19(5), 478-484.

Gonçalves, S., Lima, M., \& Marques, A. (2006). Posttraumatic stress disorder, depression and anxiety in work accident victims and witnesses: preliminary results. In C. Guedes Soares \& E. Zio (Eds.) Safety and Reliability for Managing Risk (vol. 1, pp. 767- 771). London, England: Taylor \& Francis.

Hafstad, G., Gil Rivas, V., Kilmer, R., \& Raeder, S. (2010). Parental adjustment, family functioning, and posttraumatic growth among Norwegian children and adolescents following a natural disaster. American Journal of Orthopsychiatry, 80(2), 248-257. doi: 10.1111/j.1939-0025.2010.01028.x

Helgeson, V., Reynolds, K., \& Tomich, P. (2006). A meta-analytic review of benefit finding and growth. Journal of Consulting and Clinical Psychology, 74(5), 797-816. doi: 10.1037/0022-006X.74.5.797

Hu, L., \& Bentler, P. (1999). Cutoff criteria for fit indexes in covariance structure analysis: Conventional criteria versus new alternatives. Structural Equation Modeling, 6(1), 1-55. doi: 10.1080/10705519909540118

Jiménez, N., \& Alvear, M. (2005). Accidentes de trabajo: Un perfil general. Revista de la Facultad de Medicina UNAM, 48(4), 139-144.

Joormann, J., \& Gotlib, I. H. (2010). Emotion regulation in depression: Relation to cognitive inhibition. Cognition \& Emotion, 24(2), 281-298. doi: $10.1080 / 02699930903407948$

Kilmer, R. P., \& Gil-Rivas, V. (2010). Exploring posttraumatic growth in children impacted by Hurricane Katrina: Correlates of the phenomenon and developmental considerations. Child Development, 81(4), 1211-1227. doi: 10.1111/j.14678624.2010.01463.x

Kirschenbaum, A., Oigenblick, L., \& Goldberg, A. I. (2000). Well being, work environment and work accidents. Social Science \& Medicine, 50(5), 631-639. doi: 10.1016/S02779536(99)00309-3

Koenig, H. G., Pargament, K. I., \& Nielsen, J. (1998). Religious coping and health status in medically ill hospitalized older adults. The Journal of Nervous and Mental Disease, 186(9), 513-521. doi: 10.1097/00005053-199809000-00001

Kuoppala, J., Lamminpää, A., Väänänen-Tomppo, I., \& Hinkka, K. (2011). Employee well-being and sick leave, occupational accident, and disability pension: A cohort study of civil servants. Journal of Occupational and Environmental Medicine, 53(6), 633-640. doi: 10.1097/jom.0b013e31821aa48c

Lazarus, R. S., \& Folkman, S. (1984). Stress, appraisal, and coping. New York: Springer.

Leal, F., Carmona, M., \& Ferrer, R. (2016). Rumination in posttraumatic stress and growth after a natural disaster: a model from northern Chile 2014 earthquakes. European Journal of Psychotraumatology, 7(1), 31638. doi 10.3402/ejpt.v7.31638 
Leiva-Bianchi, M., \& Gallardo, I. (2013). Validation of the Short Posttraumatic Stress Disorder Rating Interview (SPRINT-E) in a sample of people affected by F-27 Chilean earthquake and tsunami. Anales de Psicología, 29(2), 328-334. doi: 10.6018/ analesps.29.2.130681

Linley, P. A., \& Joseph, S. (2004). Positive change following trauma and adversity: A review. Journal of Traumatic Stress, 17(1), 11-21. doi: 10.1023/b:jots.0000014671.27856.7e

McConnell, K. M., Pargament, K. I., Ellison, C. G., \& Flannelly, K. J. (2006). Examining the links between spiritual struggles and symptoms of psychopathology in a national sample. Journal of Clinical Psychology, 62(12), 1469-1484. doi: 10.1002/ jclp. 20325

Morán, C., Landeros, R., \& González, M. (2010). Cope-28: un análisis psicométrico de la versión en español de Brief Cope [COPE-28: A psychometric analysis of the Spanish version of the Brief COPE]. Universitas Psychologicas, 9(2), 543-552.

Morris, B., \& Shakespeare-Finch, J. (2011). Rumination, posttraumatic growth, and distress: Structural equation modelling with cancer survivors. Psycho-Oncology, 20(11), 11761183. doi: $10.1002 /$ pon.1827

Morris, B. A., Shakespeare-Finch, J., Rieck, M., \& Newbery, J. (2005). Multidimensional nature of posttraumatic growth in an Australian population. Journal of Traumatic Stress, 18(5), 575-585. doi: 10.1002/jts.20067

Nyberg, E., Stieglitz, R. D., Frommberger, U., \& Berger, M. (2003). Psychological disorders after severe occupational accidents. Versicherungsmedizin, 55(2), 76-81.

O'Connor, D. (2017). The role of repetitive thinking and spirituality in the development of posttraumatic growth and symptoms of posttraumatic stress disorder. (Doctoral dissertation). University of Liverpool, England.

Pargament, K. I. (1997). The psychology of religion and coping: Theory, research, practice. New York: Guilford Press.

Pargament, K. I., Koenig, H. G., Tarakeshwar, N., \& Hahn, J. (2004). Religious coping methods as predictors of psychological, physical, and spiritual outcomes among medically ill elderly patients: A two-year longitudinal study. Journal of Health Psychology, 9(6), 713-730. doi: 10.1177/1359105304045366

Pargament, K., Smith, B., Koenig, H., \& Perez, L. (1998). Patterns of positive and negative religious coping with major life stressors. Journal for the Scientific Study of Religion, 37(4), 710-724. doi: 10.2307/1388152

Prati, G., \& Pietrantoni, L. (2009). Optimism, social support, and coping strategies as factors contributing to posttraumatic growth: A meta-analysis. Journal of Loss and Trauma, 14(5), 364-388. doi: 10.1080/15325020902724271

Rajandram, R., Jenewein, J., McGrath, C., \& Zwahlen, R. A. (2011). Coping processes relevant to posttraumatic growth: An evidence-based review. Support Care Cancer, 19(5), 583-589. doi: 10.1007/s00520-011-1105-0 
Rivera-Ledesma, A., \& Montero, L. (2005). Espiritualidad y religiosidad en adultos mayores mexicanos. Salud Mental, 28(6), 51-58.

Schönenberg, M., Reichwald, U., Domes, G., Badke, A., \& Hautzinger, M. (2008). Ketamine aggravates symptoms of acute stress disorder in a naturalistic sample of accident victims. Journal of Psychopharmacology, 22(5), 493-497. doi: $10.1177 / 0269881107082481$

Shaw, A., Joseph, S., \& Linley, P. A. (2005). Religion, spirituality, and posttraumatic growth: A systematic review. Mental Health, Religion \& Culture, 8(1), 1-11. doi: 10.1080/1367467032000157981

Soo, H., \& Sherman, K. A. (2015). Rumination, psychological distress and post-traumatic growth in women diagnosed with breast cancer. Psycho-Oncology, 24(1), 70-79. doi: 10.1002/pon.3596

Souto, L., De Oliveira, B., \& Haag, C. (2012). Reacciones postraumáticas en los adultos: ¿Cómo, por qué y qué aspectos a evaluar? Temas en Psicología, 20(2), 459-478.

Stockton, H., Hunt, N., \& Joseph, S. (2011). Cognitive processing, rumination, and posttraumatic growth. Journal of Traumatic Stress, 24(1), 85-92. doi: 10.1002/ jts.20606

Superintendencia de Seguridad Social. (2017). Informe Anual: Estadísticas sobre seguridad social 2016. Santiago, Chile: SUSESO. Recuperado de http://www.suseso.cl/607/ articles-40371_archivo_01.pdf

Taku, K., Cann, A., Tedeschi, R. G., \& Calhoun, L. G. (2009). Intrusive versus deliberate rumination in posttraumatic growth across US and Japanese samples. Anxiety, Stress \& Coping, 2(22), 29-36. doi: 10.1080/10615800802317841

Tedeschi, R. G., \& Calhoun, L. G. (1996). The Posttraumatic Growth Inventory: Measuring the positive legacy of trauma. Journal of Traumatic Stress, 9(3), 455-471. doi: $10.1007 / \mathrm{bf} 02103658$

Tedeschi, R. G., \& Calhoun, L. G. (2004). Posttraumatic growth: Conceptual foundations and empirical evidence. Psychological Inquiry, 15(1), 1-18. doi: 10.1207/ s15327965pli1501_01

Treynor, W., Gonzalez, R., \& Nolen-Hoeksema, S. (2003). Rumination reconsidered: A psychometric analysis. Cognitive Therapy and Research, 27(3), 247-259. doi: 10.1023/A:102391031556

Triplett, K. N., Tedeschi, R. G., Cann, A., Calhoun, L. G., \& Reeve, C. L. (2012). Posttraumatic growth, meaning in life, and life satisfaction in response to trauma. Psychological Trauma: Theory, Research, Practice, and Policy, 4(4), 400-410. doi: 10.1037/ a0024204

Vázquez, C., Castilla, C., \& Hervás, G. (2009). Reacciones ante el trauma: Resistencia y crecimiento. En E. Fernández-Abascal (Ed.), Las Emociones Positivas (pp. 375-392). Madrid, España: Pirámide. 
Vicente-Herrero, M. T., Torres, J. I., Ramírez, M. V., Terradillos, M. J., Capdevila, L., \& López-González, A. (2014). Trastorno de estrés postraumático de causa laboral: Determinación de la contingencia en España. Revista Mexicana de Salud en el Trabajo, 6(16), 56-61.

Yu, C. (2002). Evaluating cutoff criteria of model fit indices for latent variable models with binary and continuous outcomes. (Doctoral Dissertation). University of California, Los Angeles, USA. Retrieved from http://www.statmodel.com/download/Yudissertation.pdf

Zoellner, T., \& Maercker, A. (2006). Posttraumatic growth in clinical psychology: A critical review and introduction of a two component model. Clinical psychology review, 26(5), 626-653. doi: 10.1016/j.cpr.2006.01.008 